\title{
A Piston Finger Device for Restoring the Motor Function of Chronic Plegic Fingers: Analysis of the Piston Finger Technique
}

\author{
Mengsu Wang \\ Department of Electrical and Mechanical Engineering, Nagoya Institute of Technology, \\ Gokiso-cho, Syouwa-ku, Nagoya, Aichi 466-8555, Japan \\ Hirofumi Tanabe \\ Department of Health Sciences, Shonan University of Medical Sciences, \\ 16-48 Kamishinano, Totsuka-ku, Yokohama, Kanagawa 244-0806, Japan \\ Kenji Ooka \\ Department of Electrical and Mechanical Engineering, Nagoya Institute of Technology, \\ Gokiso-cho, Syouwa-ku, Nagoya, Aichi 466-8555, Japan \\ Yoshifumi Morita \\ Department of Electrical and Mechanical Engineering, Nagoya Institute of Technology, \\ Gokiso-cho, Syouwa-ku, Nagoya, Aichi 466-8555, Japan
}

E-mail:m.wang.188@nitech.jp,hirofumi.tanabe@sums.ac.jp,k.oooka.446@nitech.jp,morita@nitech.ac.jp http://www.nitech.ac.jp/eng/index.html

\begin{abstract}
A piston finger technique (PFT) was developed for restoring the motor function of chronic plegic fingers, with good treatment outcomes, including reduced spasticity and improved muscle shortening. Before developing a piston finger device (PFD) simulating the PFT, we analyzed the PFT by using a motion capture system. The motion of a chronic plegic index finger was investigated after administering two types of PFT-based treatment. The ranges of motion of the proximal interphalangeal and metacarpophalangeal joints of the index finger, and the vibration width and frequency during treatment were measured. These results will be useful for developing a PFD.
\end{abstract}

Keywords: Piston finger technique, Plegic finger, Spasticity, Motion analysis, Piston finger device

\section{Introduction}

The number of patients with cerebrovascular disease in Japan in 2014 has reached 1.179 million. ${ }^{1}$ The main sequelae of cerebrovascular disease is hemiplegia, and approximately $80 \%$ of patients with chronic hemiplegia have chronic finger paralysis. Among the symptoms of hemiplegia, the decreased motor function of the fingers is the most difficult to recover. Owing to hand paralysis, nursing care is needed in many situations in daily life and many patients are forced to give up self-sustaining living.

In hand rehabilitation therapy, the therapist performs treatment by moving the patient's fingers passively. However, because of the lack of therapists, many patients do not receive adequate treatment. In view of this situation, we aimed to develop a rehabilitation device to support therapists. 
Currently, in Japan, a treatment method has been used for therapeutic stimulation and motion reconstruction of paralyzed limb according to upper motor neuron disorders such as stroke and spinal cord injury by therapeutic electrical stimulation and functional electrical stimulation. ${ }^{2}$ The hybrid assistive neuromuscular dynamic stimulation (HANDS) therapy ${ }^{3}$ has been devised to encourage brain plastic change in chronic stroke hemiplegia patients and to improve upper limb motor function and the quality of daily life. In the development of equipment supporting finger rehabilitation, a perception support system has been developed that allows biofeedback transfer from the paralyzed side motion to the non-paralyzed side so that awareness can be directed to the paralyzed side movement sensation through the non-paralyzed side. ${ }^{4}$ In addition, a new spasticity suppression method named direct application of vibratory stimuli (DAVS) ${ }^{5}$ using vibration stimulation has been devised, which has antispastic effects in the hemiplegic upper limbs of poststroke patients.

In other countries, the constraint-induced movement therapy (CIMT) ${ }^{6}$ has been developed, to increase the functional use of the neurologically weaker upper extremity through massed practice while restraining the lesser involved upper extremity. A robotic interface for training hand and finger functions has been developed. It is a cable-actuated rehabilitation system where each finger is attached to an instrumented cable loop that allows force control and a predominantly linear displacement. ${ }^{7}$

However, only a few studies have been conducted on the validation and motion analysis of manual treatment methods. Moreover, only few research studies have been conducted to develop rehabilitation devices simulating the treatment method.

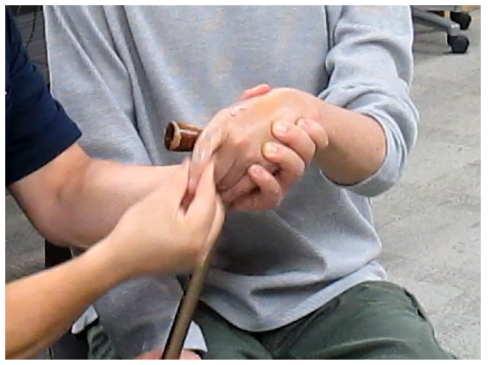

Fig. 1. Treatment with piston finger technique.
On the other hand, the second author developed a piston finger technique (PFT) for restoring the motor function of chronic plegic fingers and has achieved good treatment outcomes, including reduced spasticity and improved muscle shortening. ${ }^{8}$ The PFT is treatment for the purpose of temporary reduction of spastic paralysis, which is used as a preparatory movement before motor function restoration training.

In this study, we performed myoelectric measurement to demonstrate the effectiveness of PFT for a paralyzed index finger. We also analyzed the finger motion with the PFT by using a motion capture system to clarify the finger joint angles and the frequency during the repeated motion by PFT. Based on the analysis results, the specification of piston finger device (PFD) was determined.

\section{PFT and Its Treatment Effect}

\subsection{What is PFT?}

In general, when stretching treatment is applied to fingers with spasticity, the spastic muscle is temporarily improved. However, the spasticity often occurs again when the patient bends the affected finger. This is one of the troubles of many therapists engaged in the treatment of hemiplegia.

PFT is a technique for improving adhesion of soft tissues and spasticity. In the PFT, the therapist quickly repeats flexion and extension of the patient's finger joints. The PFT can decrease flexor spasticity in a short time and improve muscle contraction. The effect lasts longer than that by stretching treatment. Treatment with the PFT by the second author is shown in Fig.1. and the PFT has two patterns which is shown in Fig. 2. ${ }^{8}$

In Pattern 1, the therapist, with one hand, holds and

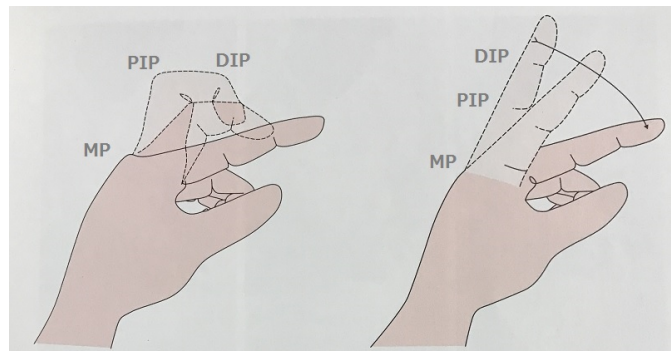

(a) Pattern 1

(b) Pattern 2

Fig. 2. Finger motion by piston finger technique ${ }^{8}$. 
fixes the patient's plegic thumb with full abduction, and with the other hand, holds and moves the distal phalange of the patient's index finger. From the start positions, the metacarpophalangeal (MP) and proximal interphalangeal (PIP) joints are bended and extended, respectively. Then, the metacarpophalangeal (MP) joint is bended and extended quickly and repeatedly, while the proximal interphalangeal (PIP) joint is extended and bended quickly and repeatedly. PFT treatment is applied to the index, middle, and ring fingers individually or together. After PFT treatment with Pattern 1, the flexor spasticity of the four fingers diminishes.

In Pattern 2, the start positions of the MP, PIP, and distal interphalangeal (DIP) joints are extended fully. Then, the MP joint is bended and extended quickly and repeatedly, while keeping the DIP and PIP joints in the extended position. After PFT treatment with Pattern 2 the MP joints of four fingers can bend to the vicinity of the end feel within the range without feeling pain.

\subsection{Treatment effect of PFT}

To verify the therapeutic effect of the PFT treatment, the therapist applied the PFT treatment to the paralyzed index finger of a male adult in his forties. The paralytic side was the left. Nine years had passed since the onset of stroke.

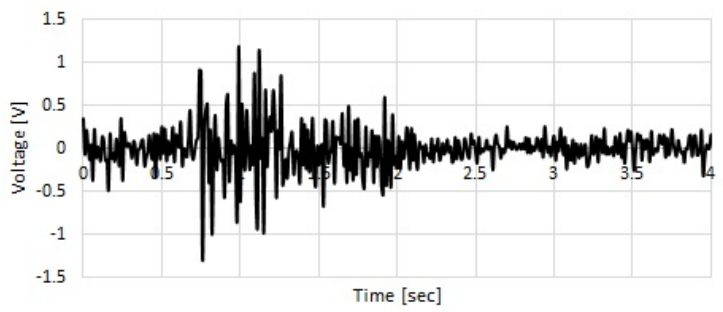

(a) Before PFT treatment.

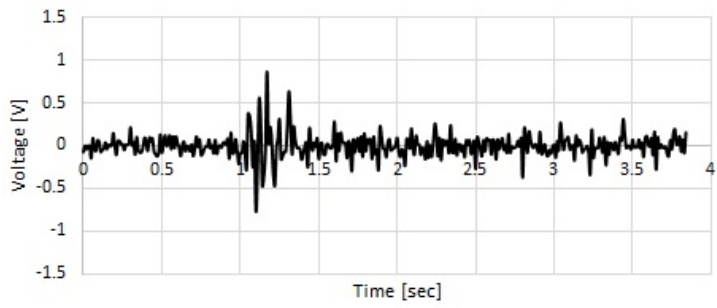

(b) After PFT treatment

Fig. 3. Electromyograms of the flexor digitorum superficialis muscle of a plegic index finger.
After treatment, the Modified Ashworth Scale (MAS) scores of the extension of the patient's plegic index finger increased from 1 to 4 . This means that spastic paralysis was reduced. Electromyograms of the flexor digitorum superficialis muscle of the patient's plegic index finger before and after treatment are shown in Fig. 3. Figure 3 shows that the abnormal muscle tone of the paralyzed finger during MAS testing by the therapist. The abnormal muscle tone was markedly reduced after PFT treatment. This means that spasticity was reduced by PFT and that the therapeutic effect was confirmed.

\section{Analysis of the PFT}

We analyzed the patient's finger motion during PFT treatment by using the VICON motion capture system (Vicon Motion Systems Ltd.).

\subsection{Motion measurement}

In Pattern 1, as the DIP joint was kept fully extended, we measured the PIP and MP joint angles of the index finger as shown in Fig. 4(a). Six markers consisting of two markers between the DIP and PIP joints, two markers between the PIP and MP joints, and two markers between the MP joint and the wrist were attached on the center line of the middle phalanges, proximal phalanges, and metacarpals of the index finger, respectively. Vectors $\boldsymbol{v}_{11}, \boldsymbol{v}_{12}, \boldsymbol{v}_{13}$, and $\boldsymbol{v}_{14}$ were defined as shown in Fig. 4(a).

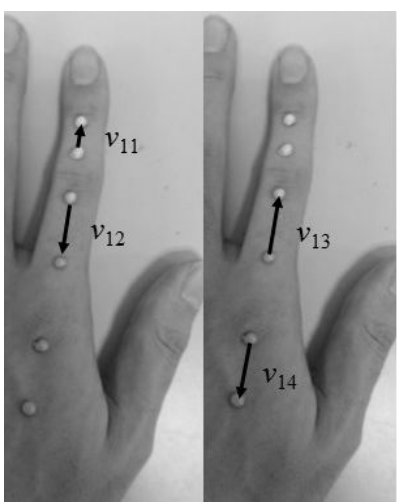

(a) Pattern 1

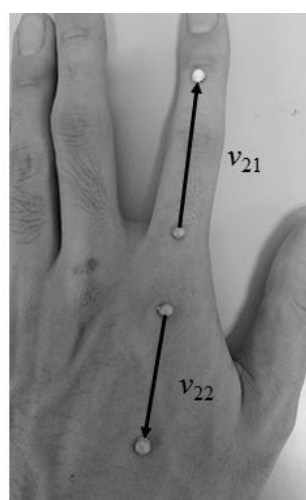

(b) Pattern 2
Fig. 4. Marker attachment positions and vectors defined for computation of joint angles. 
In Pattern 2, as the DIP and PIP joints were kept fully extended, we measured only the MP joint angle of the index finger as shown in the Fig. 4(b). Four markers consisting of one marker between the DIP and PIP joints, one marker between the PIP and MP joints, and two markers between the MP joint and the wrist were attached on the center line of the middle phalanges, proximal phalanges, and metacarpals of the index finger, respectively. Vectors $\boldsymbol{v}_{22}$ and $\boldsymbol{v}_{23}$ were defined as shown in Fig. 4(b).

\subsection{Computation of joint angles}

We computed the joint angles of the index finger during treatment. For this purpose, we measured the threedimensional coordinates of the markers with VICON and computed the joint angles from the vectors shown in Fig. 4 (a) and (b). The PIP and MP joint angles were defined as shown in Fig. 5.

In Pattern 1, the PIP and MP joint angles were denoted by $\theta_{\mathrm{PIP} 1}$ and $\theta_{\mathrm{MP} 1}$, respectively. The angles were computed by applying vectors $\boldsymbol{v}_{11}, \boldsymbol{v}_{12}, \boldsymbol{v}_{13}$, and $\boldsymbol{v}_{14}$ to the following equations:

$$
\theta_{\mathrm{PIP} 1}=\cos ^{-1} \frac{\boldsymbol{v}_{11} \cdot \boldsymbol{v}_{12}}{\left|\boldsymbol{v}_{11}\right|\left|\boldsymbol{v}_{12}\right|}, \theta_{\mathrm{MP} 1}=\cos ^{-1} \frac{\boldsymbol{v}_{13} \cdot \boldsymbol{v}_{14}}{\left|\boldsymbol{v}_{13}\right|\left|\boldsymbol{v}_{14}\right|} \text {. }
$$

In Pattern 2, the MP joint angle was denoted by $\theta_{\mathrm{MP} 2}$. The angle was computed by applying vectors $\boldsymbol{v}_{22}$ and $\boldsymbol{v}_{23}$ to the following equation:

$$
\theta_{\mathrm{MP2}}=\cos ^{-1} \frac{\boldsymbol{v}_{21} \cdot \boldsymbol{v}_{22}}{\left|\boldsymbol{v}_{21}\right| \boldsymbol{v}_{22} \mid} \text {. }
$$



Fig. 5. MP and PIP joint angles.

\section{Analysis of the Results of the PFT}

We analyzed the patient's finger motion during treatment by the therapist for 10 seconds. The time histories of the PIP and MP joint angles during treatment of Patterns 1 and 2 are shown in Figs. 6 to 8 . The summaries of the mean, maximum, and minimum values of the joint angles at the start and end positions of Patterns 1 and 2 are shown in Table 1.

In Pattern 1, we analyzed the joint angles in Figs. 6 and 7 from 2 to $10 \mathrm{sec}$. We found that the PIP joint angle was moved by the therapist between $86.7^{\circ}$ and $155.4^{\circ}$, and the MP joint angle was moved between $140.4^{\circ}$ and $172.6^{\circ}$. Moreover, the frequency of the repeated motion was found to be $4.3 \mathrm{~Hz}$. From the results of three dimensional motion analysis of flexion and extension of MP and PIP joints, we found that the motion of the fingertip can be approximated as linear motion. The mean value of the vibration width of the fingertip was $30 \mathrm{~mm}$.

In Pattern 2, we analyzed the joint angle in Fig. 8 from 0 to $10 \mathrm{sec}$. We found that the MP joint angle was moved between $109.5^{\circ}$ and $141.4^{\circ}$. Moreover, the frequency was found to be $3.6 \mathrm{~Hz}$. From the results of three dimensional motion analysis of flexion and extension of MP joint, we found that the motion of the fingertip can be approximated as linear motion. The

Table 1. Summaries of the joint angles in Patterns 1 and 2. (Start and End: start and end positions of the repeated motions, Diff.: difference between Start and End, $x(y-z), x$ : mean value, $y$ : maximum

\begin{tabular}{|c|c|c|c|}
\hline & & Pattern 1 (degrees) & Pattern 2 (degrees) \\
\hline \multirow{3}{*}{$\begin{array}{l}\text { PIP } \\
\text { joint } \\
\text { angle }\end{array}$} & Start & $155.4(135-178)$ & \\
\hline & End & $86.7(73-96)$ & \\
\hline & Diff. & $68.7(39-105)$ & \\
\hline \multirow{3}{*}{$\begin{array}{l}\text { MP } \\
\text { joint } \\
\text { angle }\end{array}$} & Start & $172.6(168-177)$ & $141.4(136-148)$ \\
\hline & End & $140.4(135-145)$ & $109.5(102-119)$ \\
\hline & Diff. & $32.2(23-42)$ & $31.9(17-46)$ \\
\hline
\end{tabular}
value, $z$ : minimum value)

Table 2. Required specifications of PFD.

\begin{tabular}{c|c|c}
\hline & Pattern 1 & Pattern 2 \\
\hline Stroke $(\mathrm{mm})$ & 30 & 46 \\
\hline Frequency $(\mathrm{Hz})$ & 4.3 & 3.6 \\
\hline
\end{tabular}




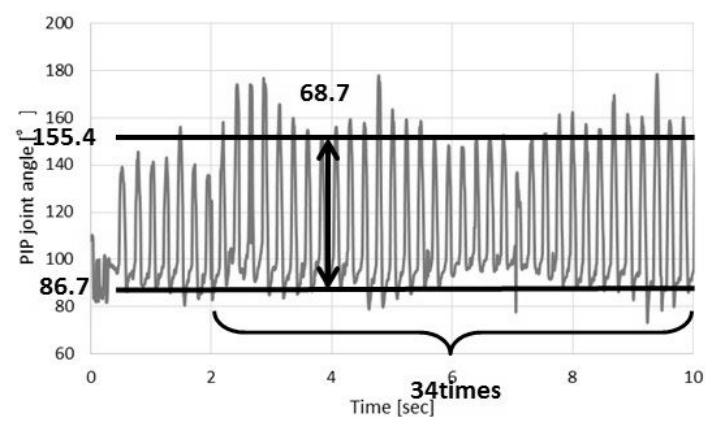

Fig. 6. Time history of PIP joint angle in Pattern 1.

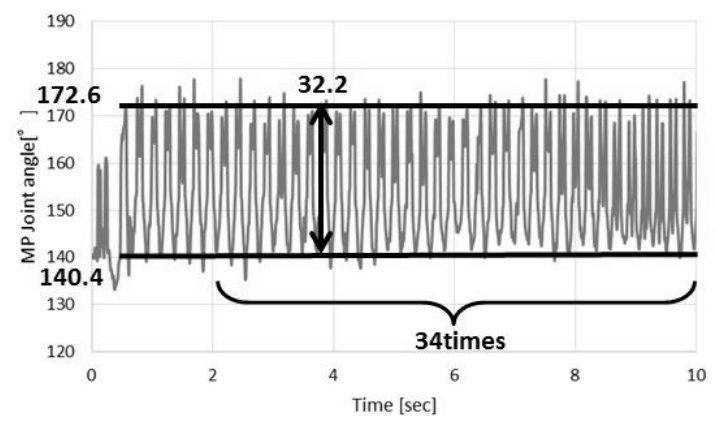

Fig. 7. Time history of MP joint angle in Pattern 1.

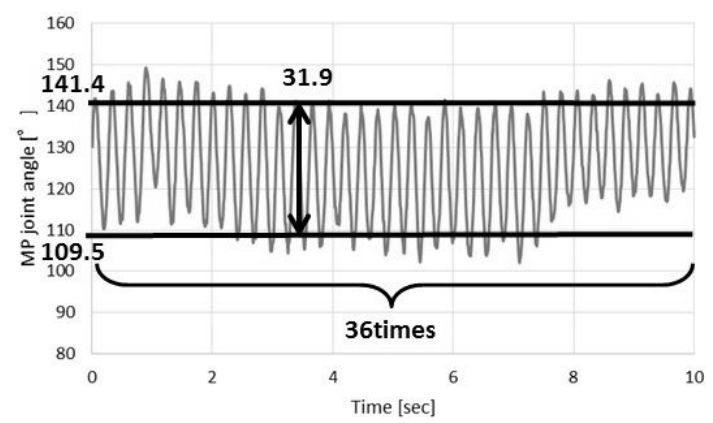

Fig. 8. Time history of MP joint angle in Pattern 2.

mean value of the vibration width of the fingertip was $46 \mathrm{~mm}$.

Based on the analysis results, we determined the specifications of PFD as shown in Table 2.

\section{Conclusion}

The therapeutic effect of PFT was confirmed by the comparison of MAS scores and electromyograms before and after treatment. The motion analysis revealed the joint angles and frequencies of finger motion during PFT treatment. Future studies are needed to develop the specifications of a PFT treatment apparatus.

\section{References}

1. Ministry of Health, Labor and Welfare, The overview of the patient survey in 2014. http://www.mhlw.go.jp/toukei/saikin/hw/kanja/14/index.h tml (in Japanese)

2. T. Matsunaga, The NESS H200 Hand Rehabilitation System for upper extremity paralysis [in Japanese], in Bull. Jpn. Soc. Prosthet. Orthot. Educ. Res. Dev., 29(2) (2013) 80-82.

3. T. Fujiwara, M. Liu, Y. Kasashima, O. Uemura, Rehabilitation for Paretic Upper Extremity of Patients with Stroke (Progress and Practice of Rehabilitation for Stroke, 43rd Annual Meeting of the Japanese Association of Rehabilitation Medicine), Jpn. J. Rehabil. Med. 43(11) (2006) 743-746.

4. H. Iwata, K. Enjyoji, R. Komagata, S. Sugano, Somatic sensation-biofeedback system for paralyzed-finger rehabilitation: development of a self-rehabilitation assisting device simultaneously cross-displaying somatic sensation of fingers [in Japanese], in Proc. the 2008 JSME Conf. on Robotics and Mechatronics, 2008, 1P1D14(1)-(2).

5. T. Noma, S. Matsumoto, M. Shimodozono, S. Etoh, K. Kawahira, Anti-spastic effects of the direct application of vibratory stimuli to the spastic muscles of hemi plegic limbs in post -stroke patients: a proof-of-principle study, J. Rehabil. Med. 44 (2012) 325-330.

6. S. L. Fritz, K. E. Light, T. S. Patterson, A. L. Behrman, S. B. Davis, Active finger extension predicts outcomes after constraint-induced movement therapy for individuals with hemiparesis after stroke, Stroke 36(6) (2005) 1172-1177.

7. L. Dovat, O. Lambercy, R. Gassert, T. Maeder, T. Milner, T. C. Leong and E. Burdet, HandCARE a cable-actuated rehabilitation system to train hand function after stroke, IEEE Trans. Neural. Syst. Rehabil. Eng. 16(6) (2008) 582-591.

8. H. Tanabe, Effective approach to reconstruct central nervous system diseases: Tanabe therapy [in Japanese]. (Human Press, Tokyo, 2016). 